\title{
Erythrocyte Lipids in Childhood
}

\author{
Robert C. Neerhout ${ }^{[37]}$ \\ Division of Hematology, Department of Pediatrics, The Gwynne Hazen Cherry Memorial Laboratory, \\ University of California School of Medicine, Los Angeles, California, USA
}

\begin{abstract}
Extract
Erythrocyte stromal lipid analysis has been performed on 26 healthy children spanning the age groups from 6 months to 14 years of age. Total lipid, lipid phosphorus and cholesterol per cell are similar in children between the ages of 6 months and 6 years. These values, in milligrams, for the $6-12$ month age group are $(4.42 \pm 0.42) \times 10^{-10}$, $(0.98 \pm 0.08) \times 10^{-11}$ and $(1.22 \pm 0.04) \times 10^{-10}$, respectively. After 6 years of age, there is an increase toward the adult values, with children in the 10-14 year age group having values, in milligrams per cell, of: total lipid, $(4.83 \pm 0.32) \times 10^{-10}$; lipid phosphorus, $(1.15 \pm 0.10) \times 10^{-11}$; cholesterol, $(1.26 \pm 0.06) \times 10^{-10}$. Changes in cell lipid content reflect primarily changes in cell size over the age period studied. In the younger age groups, the percentage of total lipid as phospholipid is lower and that as cholesterol is higher than in children over age 6 and in adults (age group 6-12 months, percentage of total lipid as phosphorus $=2.22 \pm 0.05$, that as cholesterol $=$ $27.7 \pm 3.0$; age group 10-14 yr, percentage as phosphorus $=2.39 \pm 0.17$, percentage as cholesterol $=26.1 \pm 1.8$ ). Phospholipid fractionation reveals a consistent level of sphingomyelin so that average values for all age groups studied range only from $23.9 \%$ to $24.4 \%$ of total phospholipid. Phosphatidyl choline is lowest in the youngest children studied (25.5\% in age 6-12 months), and increases to adult levels after 6 years of age (28.8\% in age group $6-10 \mathrm{yr} ; 28.2 \%$ in age group $10-14 \mathrm{yr}$ ). Consequently, the ratio of sphingomyelin: phosphatidyl choline more closely approximates the neonatal value below $6 \mathrm{yr}$ and the lower adult value after $6 \mathrm{yr}$. Fatty acids esterified to phospholipid remain relatively constant throughout the age groups studied and do not differ markedly from adult values by the end of the first year of life.
\end{abstract}

\section{Speculation}

The erythrocyte stromal lipid composition varies both quantitatively and qualitatively with the age of the subject studied. Possible pathologic changes in lipid composition should only be interpreted in the light of normal values for any given age group.

\section{Introduction}

The lipid composition of the human erythrocyte membrane has been a subject of great interest in recent years. Alterations of erythrocyte lipid composition in association with a variety of hematologic disorders as well as plasma lipoprotein disorders have been eluci- dated and extensively reviewed $[5,13,26,31]$. The influence of dietary intake of lipid on the lipid composition of erythrocytes has been studied $[7,12,21,27$, 28]. Despite this emphasis on alterations in lipid composition of erythrocytes in abnormal situations, lipid composition of the erythrocyte membrane in normal populations has been systematically studied only in the 
adult and newborn age groups $[3,6,9,14-16,18,24$, 29]. The purpose of this report is to define more clearly the expected normal lipid composition of erythrocytes from a group of unselected healthy children spanning the pediatric age range.

\section{Materials}

Blood samples were collected from 26 fasting healthy children who ranged in age from 6 months to 14 years. Selection was based solely on the willingness of the parents to cooperate in the study after they had received appropriate explanation. The subjects were either children of hospital personnel or siblings of children being followed in the University of California, Los Angeles outpatient clinic for non-familial disorders. No attempt was made to define dietary intake except to assure a state of nutrition seemingly appropriate for a middle class suburban population. All subjects had a hematocrit level that was within the accepted range of normal for age [20]. Comparison is made with previously reported studies of normal neonates and adults [16].

\section{Methods}

Venous blood samples were collected in ethylenediaminetetraacetic acid $(1 \mathrm{mg} / \mathrm{ml}$ blood) and centrifuged at $4^{\circ}$, and the plasma and buffy coat were removed. Erythrocytes were washed 2-3 times with an equal or greater volume of physiologic saline. A $60-70 \%$ suspension of erythrocytes in physiologic saline was made, and aliquots were taken for hematocrit determination and cell count [34]. Determinations of erythrocyte lipids were performed in duplicate for all study patients.

Two milliliter aliquots of erythrocyte suspension were extracted twice in chloroform-methanol $(2: 1)$ by a modification [17] of the method of Farquhar [10]. The total lipid extract was dissolved in petroleum ether and aliquots were taken for the following determinations: (1) total lipid determined gravimetrically, (2) total cholesterol [4], (3) lipid phosphorus [2], (4) phospholipid fractionation by thin layer chromatography [25] (lipid spots were localized by iodine vapor and identified by comparison with purified phospholipid standards; phosphorus content of individual fractions was determined [23]), and (5) separation of phospholipid from neutral lipid by column chromatography on silicic acid [29]. Phospholipid fatty acids were methylated [30] and quantitated by gas chromatography utilizing a 6 -ft column packed with $15 \%$ diethylene glycol succinate liquid phase on chromsorb $\mathrm{w}$ (oven temperature $=180^{\circ}$, injector temperature $=$ $280^{\circ}$, detector temperature $=210^{\circ}$ [32]. Fatty acids were identified by comparison with known standards [33] and published retention times [1]. Aldehydes were identified as described by Farquhar [11].

The coefficients of variation for major lipid fractions utilizing this analytic procedure have been reported previously [19].

\section{Results}

The results of assays for total lipid, lipid phosphorus and cholesterol per cell are shown in Figure 1. To complete the spectrum of changes with age, values for erythrocytes from cord blood as well as for adult erythrocytes are included [16]. The 26 study patients were divided into five age groups for purposes of comparison. A marked drop in erythrocyte total lipid, lipid phosphorus and cholesterol from the high values at birth is noted by six months of age. These three parameters of lipid composition of erythrocytes then re-
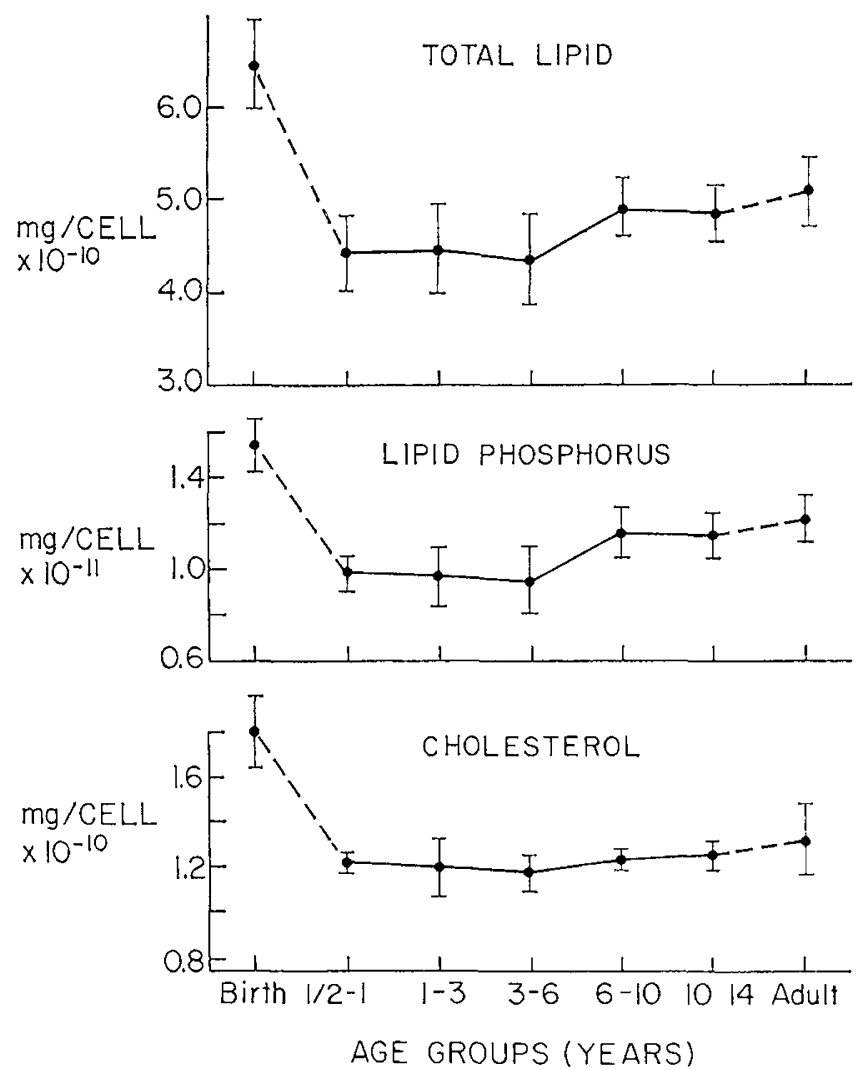

Fig. 1. Erythrocyte total lipid, lipid phosphorus (phospholipid) and cholesterol expressed as milligrams per cell for age range 6 months to 14 years. Similar values for term birth and adult red cells are included for comparison [16]. 
Table I. Lipid phosphorus (phospholipid) and cholesterol contents of total lipids of erythrocytes ${ }^{1}$

\begin{tabular}{ccc}
\hline Age, yr & Phosphorus, \% & Cholesterol, \% \\
\hline $0.5-1(3)$ & $2.22 \pm 0.05$ & $27.7 \pm 3.0$ \\
$1-3(5)$ & $2.18 \pm 0.16$ & $27.4 \pm 4.4$ \\
$3-6(4)$ & $2.16 \pm 0.14$ & $27.4 \pm 2.7$ \\
$6-10(5)$ & $2.37 \pm 0.13$ & $25.5 \pm 1.9$ \\
$10-14(9)$ & $2.39 \pm 0.17$ & $26.1 \pm 1.8$ \\
Term birth $(28)^{2}$ & $2.40 \pm 0.17$ & $27.1 \pm 2.3$ \\
Adult $(21)^{2}$ & $2.41 \pm 0.11$ & $26.0 \pm 2.1$ \\
\hline
\end{tabular}

1 Values are expressed as mean $\pm \mathrm{SD}$. Number in parentheses indicates number of subjects.

${ }^{2}$ From Neerhout [16].

main rather constant through infancy and the preschool years. Beyond the 6-yr age group, increases in erythrocyte total lipid, lipid phosphorus and cholesterol are noted. Although mean values for all three parameters in the 10-14 yr age group fall somewhat below the mean for the adult population, there is a great degree of overlap and the differences are not statistically significant.

The relative proportions of cholesterol and phospholipid in the erythrocyte membrane at the various age groups are listed in Table I. Values are expressed as percentage of total lipid as cholesterol (\% cholesterol) and percentage of total lipid as phosphorus $(\% \mathrm{P})$. As was previously noted, the $\% \mathrm{P}$ in the neonate is similar to that in the adult, whereas the \% cholesterol is slighly higher $(P<0.10)[16]$. The $\% \mathrm{P}$ has declined by 6 months of age and remains lower than the adult value through the 6-year age group $(P<$ $0.01)$. After this age, $\% \mathrm{P}$ reaches values similar to that of the adult. In comparison, \% cholesterol remains at the somewhat higher levels noted in the neonate through the 6-year period and then declines to the adult level. The variations in \% cholesterol are not significant beyond the $P<0.10$ range.
Distribution of individual phosphatides in the phospholipid fraction is tabulated in Table II. The neonate has been characterized as having a higher percentage of sphingomyelin and a lower percentage of phosphatidyl choline than the adult $[6,15,16]$. Thus the ratio of sphinogomyelin: phosphatidyl choline (S:PC) in the adult is 0.82 whereas in the neonate it approaches unity $(0.94)[15,16]$. During the age period of this study (6 months to 14 years), the sphingomyelin percentage remains quite constant and does not differ from that in the adult. The percentage of phosphatidyl choline is significantly lower than in the adult group throughout the age groups up to age 6 yr $(P<$ $0.01)$. None of the subjects who were less than 1-yr-old had a percentage of phosphatidyl choline in excess of $26 \%$, whereas no subject over age 6 yr had a percentage of phosphatidyl choline below $26.0 \%$. Three of nine subjects in the 1-6 yr group had a percentage of phosphatidyl choline below $26.0 \%$ while three had a percentage above $28.0 \%$, thus showing a period of apparent transition. Because of the low percentage of phosphatidyl choline in the children less than 1-yr-old, the $S: P C$ ratio remains high $(0.96)$ as in cord blood despite the adult level of sphingomyelin. This ratio in the 6-10 yr age group (0.84) and the 10-14 yr age group (0.85) approaches that of the adult. The combined phosphatidyl serine + phosphatidyl inositol fraction remains above the adult level throughout childhood. The percentage of phosphatidyl ethanolamine is relatively consistent throughout the age groups studied, being statistically elevated above cord blood levels only in the group under $1 \mathrm{yr}$ of age $(P<0.01)$.

The distribution of fatty acids esterified to phospholipid is presented in Table III. Nineteen fatty acids and aldehydes were quantitated but only those comprising more than $3 \%$ of the total are listed, for simplicity. It can be seen that the pattern of high palmitic

Table II. Distribution of individual phosphatides in phospholipid fraction ${ }^{1}$

\begin{tabular}{ccccccc}
\hline Age, $\mathrm{yr}$ & LL & Sph & PC & PS + PI & PE \\
\hline $0.5-1(3)^{2}$ & $1.5 \pm 0.2$ & $24.4 \pm 1.2$ & $25.5 \pm 0.5$ & $15.0 \pm 0.3$ & $33.1 \pm 0.3$ & $0.4 \pm 0.2$ \\
$1-3(5)$ & $1.6 \pm 0.4$ & $23.9 \pm 1.2$ & $27.4 \pm 1.1$ & $15.1 \pm 0.9$ & $31.4 \pm 1.0$ & $0.7 \pm 0.5$ \\
$3-6(4)$ & $1.3 \pm 0.1$ & $24.4 \pm 0.8$ & $26.7 \pm 1.7$ & $15.5 \pm 1.4$ & $31.5 \pm 0.5$ & $0.6 \pm 0.4$ \\
$6-10(5)$ & $1.1 \pm 0.3$ & $24.3 \pm 1.0$ & $28.8 \pm 1.1$ & $14.6 \pm 0.3$ & $30.5 \pm 0.9$ & $0.6 \pm 0.4$ \\
$10-14(9)$ & $1.1 \pm 0.2$ & $24.0 \pm 1.0$ & $28.2 \pm 1.6$ & $15.5 \pm 0.8$ & $30.7 \pm 1.2$ & $0.7 \pm 0.4$ \\
Term birth (28) & $1.0 \pm 0.1$ & $26.0 \pm 2.8$ & $27.7 \pm 2.1$ & $15.2 \pm 1.6$ & $29.1 \pm 2.9$ & $0.9 \pm 0.5$ \\
Adult (21) & $1.2 \pm 0.4$ & $24.1 \pm 2.0$ & $29.5 \pm 1.4$ & $13.1 \pm 1.2$ & $31.2 \pm 1.5$ & $1.0 \pm 0.2$ \\
\hline
\end{tabular}

1 Values are expressed as percentage of total lipid phosporus and each represents mean \pm sD. LL: lysolecithin, Sph : sphingomyelin, PC: phosphatidyl choline, PS : phosphatidyl serine, PI : phosphatidyl inositol, PE : phosphatidyl ethanolamine, PA: phosphatidic acid. ${ }^{2}$ Number in parentheses indicates number of subjects.

${ }^{3}$ From Neerhout [16]. 
Table. III. Distribution of fatty acids esterified to phospholipid ${ }^{1}$

\begin{tabular}{|c|c|c|c|c|c|c|c|}
\hline Age, yr & $16: 0$ & 18:0 & $18: 1$ & $18: 2$ & $20: 4$ & $22: 6$ & $24: 0$ \\
\hline $0.5-1 \quad(3)^{2}$ & $17.7 \pm 1.9$ & $16.7 \pm 0.3$ & $13.2 \pm 1.1$ & $8.8 \pm 2.7$ & $17.3 \pm 2.2$ & $4.0 \pm 0.7$ & $4.2 \pm 0.2$ \\
\hline $1-3 \quad(5)$ & $17.4 \pm 1.4$ & $17.2 \pm 1.4$ & $14.1 \pm 1.1$ & $8.7 \pm 1.1$ & $17.0 \pm 0.8$ & $4.3 \pm 0.7$ & $3.6 \pm 0.5$ \\
\hline $3-6(4)$ & $17.8 \pm 1.0$ & $17.5 \pm 1.3$ & $16.6 \pm 0.6$ & $9.9 \pm 1.2$ & $16.1 \pm 1.2$ & $3.1 \pm 0.5$ & $3.8 \pm 0.5$ \\
\hline $6-10(5)$ & $18.1 \pm 1.3$ & $16.8 \pm 0.8$ & $15.4 \pm 0.6$ & $10.4 \pm 0.9$ & $15.9 \pm 1.5$ & $3.8 \pm 1.0$ & $3.6 \pm 0.4$ \\
\hline $10-14 \quad(9)$ & $19.2 \pm 3.8$ & $16.4 \pm 0.8$ & $15.5 \pm 1.1$ & $9.9 \pm 1.6$ & $15.5 \pm 1.5$ & $3.7 \pm 1.0$ & $3.5 \pm 0.4$ \\
\hline Term birth $(28)^{3}$ & $21.3 \pm 2.4$ & $16.3 \pm 1.0$ & $11.9 \pm 1.1$ & $3.4 \pm 0.6$ & $19.6 \pm 1.4$ & $6.6 \pm 1.1$ & $5.5 \pm 1.6$ \\
\hline Adult $(21)^{3}$ & $17.0 \pm 1.2$ & $15.3 \pm 0.2$ & $14.6 \pm 1.0$ & $10.9 \pm 1.2$ & $17.4 \pm 1.1$ & $4.8 \pm 0.8$ & $4.9 \pm 1.4$ \\
\hline
\end{tabular}

1 Values are expressed as percentage of total fatty acids by weight and each represents mean \pm sD. Fatty acids are designated by carbon chain length:number of double bonds.

2 Number in parentheses indicates number of subjects.

From Neerhout [16].

acid (16:0) and arachidonic acid (20:4) coupled with lower levels of oleic acid (18:1) and linoleic acid (18:2) which distinguishes the neonate from the adult is largely lost by the earliest period of this study (6 months). The longer chain fatty acids (C22, C24) fall below the values for either the normal neonate or the adult throughout childhood.

\section{Discussion}

The subjects included in this study were chosen to be a representative spectrum of normal healthy children spanning the age group from 6 months to 14 years. All subjects in this study were ingesting whole cow's milk in addition to the appropriate solid food diet for age. Consequently it was felt that these data could be considered representative for normal children on what is loosely referred to as a "regular diet for age".

The most striking finding in this study is the low values for total lipid, lipid phosphorus and cholesterol per cell noted in the age group 6 months -6 years. This is in marked contrast to the elevated levels noted in term neonates as compared with normal adults $[6,16]$. Since lipid in erythrocytes is limited to the cell membrane [8], it has been generally concluded that variations in cell membrane surface area will be reflected by variations in cell membrane lipid content [5]. Since cell surface area is difficult to determine accurately, previous discussions have utilized the association between lipid content of erythrocytes and volume of erythrocytes to express this relation $[16,18,19]$. Thus, the normal newborn erythrocyte has $5.38 \times 10^{-10} \mathrm{mg}$ lipid/100 $\mu^{3}$ while the normal adult ranges from 5.07 to $5.14 \times 10^{-10} \mathrm{mg}$ lipid $/ 100 \mu^{3}$ [19]. Utilizing such calculations, it can be shown that the variations in lipid content of erythrocytes noted in this study largely reflect the variations in mean corpuscular volume of erythrocytes in the respective age groups. The mean corpuscular volume of the saline washed cells in the 6 month -6 year groups is $88 \mu^{3}$ with $5.08 \mathrm{mg}$ lipid $/ 100 \mu^{3}$ volume. The combined 6-14 year group has a mean corpuscular volume of $92.5 \mu^{3}$ with $5.17 \mathrm{mg}$ lipid/100 $\mu^{3}$. Thus the relation between erythrocyte membrane lipid and erythrocyte volume throughout the age groups of this study is similar to that noted in adult cells. The relation of total lipid to erythrocyte volume noted in the neonate $\left(5.38 \times 10^{-10} \mathrm{mg} / 100 \mu^{3}\right)$ remains, therefore, unique to that group. A progressive decline in mean corpuscular volume following birth has been well shown and it suggests a selective loss of the more macrocytic cell population in the first few weeks of life [22]. The quantitative differences in lipid composition between erythrocytes of the neonate and those of older children would appear to be largely associated with the loss of this macrocytic cell population.

The explanation for the higher sphingomyelin:phosphatidyl choline ratio noted at birth and up to 6 years of age remains obscure. Previous investigators have reasoned that the $S: P C$ ratio is higher in the neonate than in the adult because the $S: P C$ ratio in plasma is higher in the neonate $[6,15]$. Plasma phospholipid analysis was performed on 7 of the samples from the 6-14 year age group and revealed that phosphatidyl choline comprises $67.9 \%$ and sphingomyelin $16.5 \%$ of the total phospholipid. Previous studies from our laboratory revealed rather similar values for cord blood (phosphatidyl choline $=67.6 \%$, sphingomyelin 17.9\%) [19]. Thus the S:PC ratio in plasma in the $6-14$ year group is 0.24 whereas that in cord blood is 0.26 . Although the ratios differ in a manner similar to that for the erythrocyte, the difference in the $S: P C$ ratio in plasma is not statistically significant. Whether this seemingly minor variation in phospholipid distri- 
bution in plasma is, in fact, the cause of the lipid variation in erythrocytes must await further investigation. Numerous human and animal studies have shown that wide variation in lipid composition of the diet does not affect the proportions of the major phospholipid classes of the erythrocyte [7, 12, 27].

Analysis of the fatty acids esterified to phospholipid show that the major variations which distinguish cord blood cells from adult cells have largely disappeared by the end of the first year of life. The most striking change is the increased percentage of linoleic acid (18:2) already present by the end of the first year. Controlled dietary studies have shown that the content of linoleic acid in erythrocytes closely follows the linoleic acid content of the diet $[7,12,28]$. Changes in dietary linoleic acid content are reflected in similar changes in the erythrocyte within a few weeks [12]. The consistency of the values for erythrocyte linoleic acid content throughout the span of the age groups in this study suggests therefore a fairly consistent intake of this dietary lipid by this unselected population.

\section{Summary}

Data are presented that delineate the erythrocyte stromal lipid composition in the age group of 6 months to 14 years. Variations in total cell lipid tend to parallel the changes in erythrocyte volume and, presumably, erythrocyte surface area. The percentage of total lipid which is phospholipid is lower in young children and reaches adult levels after 6 years of age. Quantitation of individual phosphatides reveals a consistent level of sphingomyelin throughout the study age groups. An increase in percentage of phosphatidyl choline after age $6 \mathrm{yr}$ leads to a sphingomyelin:phosphatidyl choline ratio similar to that of the adult, in contrast to a higher value noted in younger children and in cord blood. The phospholipid fatty acid pattern characteristic of cord blood (higher levels of palmitic and arachidonic acids, lower levels of oleic and linoleic acids than in the adult) is largely gone by the earliest period of this study. In particular, linoleic acid levels approach those of the older child and the adult by the end of the first year of life.

\section{References and Notes}

1. Ackman, R. G.: Structural correlation of unsaturated fatty acid esters through graphical comparison of gas-liquid chromatographic retention times on a polyester substrate. J. Amer. Oil Chem. Soc., 40: 558 (1963).

2. Bartlett, G. R.: Phosphorus assay in column chromatography. J. Biol. Chem., 234: 466 (1959).
3. Bentlex, H. P., JR.: Erythrocyte phospholipids in the newborn infant. Proc. Soc. Exp. Biol., 111: 591 (1962).

4. Chiamori, N., and Henry, R. J.: Study of the ferric chloride method for determination of total cholesterol and cholesterol esters. Amer. J. Clin. Path., 31: 305 (1959).

5. COOPER, R. A.: Lipids of human red cell membrane: normal composition and variability in disease. Sem. Hemat., 7: 296 (1970).

6. Crowley, J., Ways, P., And Jones, J. W.: Human fetal erythrocyte and plasma lipids. J. Clin. Invest., 44: 989 (1965).

7. DE Gier, J., AND van DeENEN, L. L. M.: A dietary investigation on the variations in phospholipid characteristics of red-cell membranes. Biochim. Biophys. Acta, 84: 294 (1964).

8. Dodge, J. T., Mrtchell, C., and Hanahan, D. J.: The preparation and chemical characteristics of hemoglobin-free ghosts of human erythrocytes. Arch. Biochem. Biophys., 180: 119 (1963).

9. Dodge, J. T., And Phillres, G. B.: Composition of phospholipids and of phospholipid fatty acids and aldehydes in human red cells. J. Lipid Res., 8: 667 (1967).

10. Farquhar, J. W.: Human erythrocyte phosphoglycerides. I. Quantification of plasmalogens, fatty acids and fatty aldehydes. Biochim. Biophys. Acta, 60: 80 (1962).

11. Farquhar, J. W.: Identification and gas-liquid chromatographic behavior of plasmalogen aldehydes and their acetal, alcohol and acetylated alcohol derivatives. J. Lipid Res., 3: 21 (1962).

12. Farquhar, J. W., and Ahrens, E. H., Jr.: Effects of dietary fats on human erythrocyte fatty acid patterns. J. Clin. Invest., 42: 675 (1963).

13. Hollán, S. R., and Breuer, J. H.: On red cell lipids. Acta Med. Acad. Scient. Hung., 25: 421 (1968).

14. Hollán, S. R., Szelényx, J. G., Breuer, J. H., Medgyesi, G. A., AND Sörér, V. N.: Structural and functional differences between human foetal and adult exythrocytes. Haematologia, 4: 409 (1967).

15. Hürter, P., Schröter, W., Schedel, I., ANd Gercken, G.: Phospholipids of red cell and blood plasma in adults, newborn infants, and patients with $\mathrm{Rh}$ erythroblastosis. Pediatrics, $46: 259$ (1970).

16. Neerhout, R. C.: Erythrocyte lipids in the neonate. Pediat. Res., 2: 172 (1968).

17. Neerhour, R. C.: Abnormalities of erythrocyte stromal lipids in hepatic disease. J. Lab. Clin. Med., 71: 438 (1968).

18. Neerhout, R. C.: Erythrocyte lipids in infants with low birth weights. Pediat. Res., 5: 101 (1971).

19. Neerhout, R. C.: Alteration of adult erythrocyte lipids during in vivo fetal circulation. Pediat. Res., 5: 683 (1971).

20. Nelson, W. E., Vaughan, V. C., III, and McKay, R. J.: Textbook of Pediatrics, 9th Ed. (W. B. Saunders Co., Philadelphia, 1969).

21. Nrshimira, H.: Studies on the fatty acid composition of the erythrocyte phospholipid in childhood. Acta Paediat. Jap., 11: 22 (1969).

22. Oski, F. A., and Naiman, J. L.: Hematologic Problems in the Newborn. (W. B. Saunders Co., Philadelphia, 1966).

23. Parker, F., and Peterson, N. F.: Quantitative analysis of phospholipids and phospholipid fatty acids from silica gel thin-layer chromatography. J. Lipid Res., 6: 455 (1965).

24. Reed, C. F., Swisher, S. N., Marinettr, G. V., and Eden, E. G.: Studies of the lipids of the erythrocyte. I. Quantitative 
analysis of normal human red blood cells. J. Lab. Clin. Med., 56: 281 (1960).

25. Skrpsky, V. P., Pererson, R. F., and Barclay, M.: Separation of phosphatidylethanolamine, phosphatidylserine and other phospholipids by thin larer chromatography. J. Lipid Res., 3: 467 (1962).

26. vaN DeEnen, L. L. M., AND DE Grer, J.: Chemical composition and metabolism of lipids in red cells of various animal species. In: C. Bishop and D. M. Surgenor: The Red Blood Cell. (Academic Press, New York, 1964).

27. Walker, B. L., AND KumMerow, F. A.: Dietary fat and the structure and properties of rat exythrocytes. I. Effect of dietary fat on the erythrocyte lipids. J. Nutrit., 81: 75 (1963).

28. Walker, B. L., And Kumuerow, F. A.: Dietary fat and the structure and properties of rat erythrocytes. III. Response of erythrocyte fatty acids to various dietary fats. J. Nutrit., 82: 329 (1964).

29. WAYS, P., AND HaNAHAX, D. J.: Characterization and quantification of red cell lipids in normal man. J. Lipid Res., 5: 318 (1964).
30. Ways, P., ReEd, C. F., and Hanahan, D. J.: Red cell and plasma lipids in acanthocytosis. J. Clin. Invest., 42: 1248 (1963).

31. Zahler, P.: The structure of the erythrocyte membrane. Experentia, 25: 449 (1969).

32. Model 810, Perkin-Elmer Corp., Norwalk, Conn.

33. Applied Science Laboratories, Inc., State College, Pa.

34. Model B, Coulter Electronics, Hialcah, Fla.

35. The author is indebted to Mrs. Joan Slimacovsky for her invaluable technical assistance and to the members of the UCLA Pediatric house-staff for their cooperation in obtaining samples for study.

36. This work was supported by United States Public Hcalth Service Rescarch Grant no. 2-R01-HD02124 and the Robert Shaw Memorial Research Fund.

37. Requests for reprints should be addressed to: Robert C. Neerhout, M.D., Department of Pediatrics, UCLA School of Medicine, Los Angeles, Calif. 90024 (USA).

38. Accepted for publication May 3, 1972. 